\title{
Therapeutic Angiogenesis. The Present and the Future
}

\author{
Alexandre Cesar Ferreira
}

Miami-FL

Transfection of genes encoding angiogenic cytokines, particularly those naturally secreted by intact cells, may constitute an alternative treatment strategy for patients with extensive tissue ischemia in whom contemporary therapies (antianginal medications, angioplasty, bypass surgery) have previously failed or are not feasible. This strategy is designed to promote the development of supplemental collateral blood vessels that will constitute endogenous bypass conduits around occluded native arteries, a strategy termed "therapeutic angiogenesis" ${ }^{1}$.

Studies have suggested that 2 angiogenic growth factors, in particular basic fibroblast growth factor (FGF2) and vascular endothelial growth factor (VGEF), are sufficiently potent to merit further investigation ${ }^{2}$. The specificity of VEGF for endothelial cells, compared with the more widespread effect of other factors like fibroblast growth factor (FGF), may provide a broader therapeutic potential ${ }^{3}$. Four major forms of VEGF are generated as a result of alternative splicing of a single gene: VEGF 121, VEGF 165, VEGF 189, and VEGF 206. Most studies have been performed using the VEGF 165 isoform although recently VEGF 121 has also been receiving increasing attention.

The angiogenic effect of VEGF can be explained in several ways: by direct stimulation of proliferation and migration of endothelial cells in vivo, by recruitment of previously existing collaterals and by improving vasomotor function of vessels in ischemic tissue.

Insufficient angiogenesis may lead to therapy failure

University of Miami - From the Department of Medicine and the Division of Cardiology

Mailing address: Alexandre Cesar Ferreira - University of Miami, School of Medicine - Cardiovascular Laboratory (D-62) - P.O. Box 016960 - Miami-FL/ USA 33101

Received for publication on $12 / 1 / 01$

Accepted on $9 / 5 / 01$ and tissue ischemia. A rational design for angiogenic treatment strategies mandates a better understanding of the molecular mechanisms of angiogenesis. The angiogenic cytokine receptors transduce signals mediating endothelial cell proliferation, migration and organization into functional vessels and remodeling of the vessel network. In recent years, rapid progress has been made in understanding the receptor-ligand interactions that orchestrate the neovascularization process ${ }^{4}$. A novel interaction between the junctional protein vascular endothelial-cadherin (VEcadherin) and VEGF appears essential for the endothelial function of VEGF ${ }^{5}$. Furthermore, VEGF promotes vascular protection. Vascular protection is defined as the VEGFinduced enhancement of endothelial functions that mediate the inhibition of vascular smooth muscle cell proliferation, enhanced endothelial cell survival, suppression of thrombosis and antiinflammatory effects. VEGF-induced synthesis of nitric oxide and prostacyclin are both likely to be key mediators of VEGF-dependent vascular protection ${ }^{6}$. Investigation into vascular protection should help us gain insight into the underlying mechanisms of the cardiovascular actions of VEGF and should prove valuable in the development of novel therapeutic approaches based on local VEGF gene delivery.

The optimal preparation and delivery strategy for therapeutic neovascularization is the subject of ongoing clinical investigation. The potential requirement to maintain a suitably high, local concentration over a period of days to weeks constitutes an advantage for gene transfer versus recombinant protein therapy ${ }^{7}$. Although viral vectors may enhance transfection efficiency and thus yield higher levels of gene expression ${ }^{8}$, this is less relevant for strategies in which gene products, such as VEGF, include a signal sequence that permits active secretion from intact cells. Naked DNA that encodes for a secreted protein, as opposed to proteins that remain intracellular, can yield meaningful biological outcomes due to paracrine effects of the secreted gene product ${ }^{9}$. 
A phase 1 clinical study to determine the safety and bioactivity of direct myocardial gene transfer of vascular endothelial growth factor (VEGF) as the sole therapy for patients with symptomatic myocardial ischemia was conducted. Naked plasmid DNA encoding VEGF (phVEGF165) was injected directly into the ischemic myocardium via a mini left anterior thoracotomy in 5 patients with angina and documented coronary artery disease. All patients had significant reduction in angina (nitroglycerin use $=53.9 \pm 10.0 /$ week pretreatment versus $9.8 \pm 6.9$ /week posttreatment, $\mathrm{P}<0.03$ ). Objective evidence of reduced ischemia was documented by using dobutamine single photon emission computed tomography (SPECT)-sestamibi imaging in all patients ${ }^{10}$. This small study suggested that direct myocardial injection of naked plasmid DNA, via a minimally invasive chest wall incision, is safe and may lead to reduced symptoms and improved myocardial perfusion in select patients with chronic myocardial ischemia. Although safe, delivery of therapy required thoracotomy, with all the possible risks and morbidity associated with a surgical procedure, which underscores the need for developing less invasive routes of administration.

An initial series of in vivo experiments ${ }^{11}$ used intravenous administration of VEGF, with or without heparin, to result in both anatomic and physiologic evidence of enhanced collateral vessel formation in an animal model. At that time, single-bolus systemic administration of VEGF was felt to be a feasible therapeutic strategy. Systemic effects have been observed. Transient hypotension, due to VEGF-induced release of nitric oxide, and peripheral edema associated with an increase in vascular permeability are not uncommon systemic side effects.

Most strategies for transcatheter delivery of angiogenic factors have used an intracoronary route, which may have limitations because of imprecise localization of genes or proteins and systemic delivery to noncardiac tissue ${ }^{12}$. Catheter-based endocardial injection of angiogenic factors may provide an equivalent benefit without the need for surgery. A new guidance system for intramyocardial therapy utilizes magnetic fields and catheter-tip sensors to locate a position in space and reconstruct 3-dimensional left ventricular electromechanical maps without using fluoroscopy. This less invasive catheter-based system offers similar gene delivery efficiency and, thus, may have clear advantages compared with the surgically based transepicardial injection via thoracotomy ${ }^{13}$.

Intrapericardial delivery of angiogenic factors may offer a theoretical advantage of prolonged exposure of either coronary or myocardial tissue to the administered drug as the result of a reservoir function of the pericardium without major systemic effects ${ }^{14}$. A single intrapericardial injection of fibroblast growth factor (FGF)-2 in a porcine model of chronic myocardial ischemia resulted in functionally significant myocardial angiogenesis, without any adverse outcomes. This mode of FGF- 2 administration may prove to be a useful therapeutic strategy for treating patients with ischemic heart disease ${ }^{15}$.

Angiogenesis, however, is a two-sided coin with detrimental consequences in nontarget tissues. In particular, the theoretic risk of plaque angiogenesis must not be ignored. In particular VEGF-mediated enhancement of permeability may facilitate plaque expansion and rupture because intraplaque vessels tend to be quite permeable even in the absence of VEGF. Furthermore, one might imagine that growth factors, particularly FGF, which lacks specificity for endothelial cells, may accelerate progression of coronary disease by stimulating growth of fibroblasts and medial smooth muscle cells.

Pathologic angiogenesis is also involved in the pathogenesis of arthritis, diabetic retinopathy, and tumor growth and metastasis. Diabetic retinopathy is associated with the presence of growth factors in the vitreous humor, and administration of VEGF-neutralizing antibodies arrests the process. Administration of FGF in high doses is associated with anemia, thrombocytopenia and membranous nephropathy ${ }^{16}$.

Angiogenesis and the development of metastasis are intrinsically connected. Experimental data suggest that establishment and growth of metastasis are influenced by soluble factors secreted from the originating solid tumor. Solid tumors require a constant vascular supply. Tumorassociated neovascularization allows the tumor cells to express their critical growth advantage. Various angiogenic growth factors and cytokines induce neovascularization in tumors, namely members of the vascular endothelial growth factor (VEGF) and angiopoietin (Ang) gene families ${ }^{17}$. A strong correlation has been found between VEGF expression and increased tumor microvasculature and metastasis, mainly in breast cancer. It is feasible that a transient increase in VEGF levels could lead to rapid growth of quiescent metastasis. To date, due to concern about promoting metastasis growth, all studies using angiogenic growth factors mandated exclusion of patients with any history of prior malignancies, with the exception of cutaneous basal cell carcinoma.

Despite all the theoretical concerns with the use of growth factors, based on experimental and clinical data, every reason exists to believe that a short-term increase in circulating VEGF is safe. Clearly, if further clinical trials confirm the initial results of the preliminary human data, this new concept of improving intramyocardial blood flow with gene therapy is likely to be expanded to benefit a vast cohort of patients with clinically important coronary artery disease. 


\section{References}

1. Amant C, Berthou L, Walsh K. Angiogenesis and gene therapy in man: dream or reality. Drugs 1999; 59: 33-6.

2. Isner, JM. Therapeutic angiogenesis: a new frontier for vascular therapy. Vasc Med 1996; 1: 79-87.

3. Banga JD. Therapeutic angiogenesis through intramuscular injection of the gene for vascular endothelial growth factor. Ned Tijdschr Geneeskd 2000; 144: 113-6.

4. Veikkola T, Alitalo K. VEGF receptors and angiogenesis. Semin Cancer Biol 1999; 3 : 211-20.

5. Carmeliet P, Collen D. Molecular basis of angiogenesis: role of VGEF and VEcadherin. Ann NY Acad Sci 2000; 902: 249-62.

6. Zachary I, Mathur A, Yla-Herttula S, Martin J. A novel non-angiogenic cardiovascular role for vascular endothelial growth factor. Arterioscler Thromb Vasc Biol 2000; 20: 1512-20.

7. Isner JM. Angiogenesis: a breakthrough technology in cardiovascular medicine. J Invas Cardiol 2000; 12(Suppl A): 1A-17A.

8. Rosengart TK, Lee LY, Patel SR, et al. Angiogenesis gene therapy: phase I assessment of direct intramyocardial administration of an adenovirus vector expressing VEGF121 cDNA to individuals with clinically significant severe coronary artery disease. Circulation 1999; 100: 468-74.

9. Losordo DW, Pickermo JG, Takeshita S. Use of the rabbit ear artery serially assess foreign protein secretion after site specific arterial gene transfer in vivo: evidence that anatomic identification of successful gene transfer may underesti- mate the potential magnitude of transgene expression. Circulation 1994; 89 785-92.

10. Losordo DW, Vale PR, Symes JF, et al. Gene therapy for myocardial angiogenesis initial clinical results with direct intramyocardial injection of phVEGF 165 as sole therapy for myocardial ischemia. Circulation 1998; 98: 2800-4.

11. Bauters C, Asahara T, Zheng LP, et al. Site specific therapeutic angiogenesis after systemic administration of vascular endothelial growth factor. J Vasc Surgery 1995; 21: 314-24.

12. Kornowski R, Fuchs S, Leon MB, Epstein SE. Delivery strategies achieve therapeutic myocardial angiogenesis. Circulation 2000; 101: 454-8.

13. Vale PR, Losordo DW, Milliken CE. Isner JM. Left ventricular electromechanical mapping to assess efficacy of phVEGF 165 gene transfer for therapeutic angiogenesis in chronic myocardial ischemia: angiogenesis. Circulation 2000; 102: 965-74.

14. Laham RJ, Post M, Sellke FW, Simeans M. Therapeutic angiogenes using local perivascular and pericardial delivery. Curr Interv Cardiol Rep 2000; 2: 213-7.

15. Laham RJ, Rezaee $M$, Poest $M$, et al. Intrapericardial delivery of fibroblast growth factor -2 induces neovascularization in a porcine model of chronic ischemia. J Pharmacol Exp Ther 2000; 292: 795-802.

16. Baumgartner I. Therapeutic angiogenesis: theoretic problems using vascular endothelial growth factor. Curr Cardiol Rep 2000; 2: 24-8.

17. Saaristo A, Karpanen T, Alitalo K. Mechanisms of angiogenesis and their use in the inhibition of tumor growth and metastasis. Oncogene 2000; 199: 6122-9. 